\title{
On a multiple trigonometric series
}

\author{
by \\ M. Z. Garaev (Taipei)
}

1. Introduction. Throughout the text $c, c_{1}, \ldots, c_{5}$ denote absolute positive constants not necessarily the same in different cases. The constants implicit in the $O$ symbols are also absolute.

G. I. Arkhipov and K. I. Oskolkov [1] proved that for any polynomial $P(x)=\alpha_{1} x^{n}+\ldots+\alpha_{n} x+\alpha_{n+1}$ with real coefficients $\alpha_{1}, \ldots, \alpha_{n+1}$ the sequence

$$
H_{N}=\sum_{1 \leq|x| \leq N} \frac{e^{2 \pi i P(x)}}{x}
$$

converges as $N \rightarrow \infty$. They applied Vinogradov's method of trigonometric sums. Afterwards K. I. Oskolkov [3, 4] discovered a nontrivial application of this result and Vinogradov's method to the investigation of the properties of solutions of Schrödinger type PDEs. To PDEs with mixed derivatives correspond multiple trigonometric sums. The simplest type of such sums is $h_{N}=h_{N}(\alpha)$ defined as follows:

$$
h_{N}=\sum_{x=1}^{N} \sum_{y=1}^{N} \frac{\sin (\alpha x y)}{x y} .
$$

The question of convergence of $\left\{h_{N}\right\}_{N=1}^{\infty}$ was discussed several times by G. I. Arkhipov, V. N. Chubarikov and the author. But still it has not been known to us whether this sequence is convergent for all real $\alpha$ or not.

In this note we give an answer to this question.

2. TheOREM. There exists a real number $\alpha$ such that the sequence (1) diverges as $N \rightarrow \infty$.

We will use the following well known statement (see e.g. [2, p. 473]):

2000 Mathematics Subject Classification: Primary 11L03. 
LEMMA 1. For any real number $t$ we have

$$
\varrho(t)=\sum_{1 \leq y \leq Y} \frac{\sin (2 \pi y t)}{\pi y}+O\left(\frac{1}{1+Y|\sin \pi t|}\right)
$$

where $\varrho(t)=1 / 2-\{t\}$.

LEMMA 2. For any positive integers $x, q, N$ we have

$$
\sum_{1 \leq y \leq q N} \frac{\sin \left(2 \pi \frac{x y}{N}\right)}{y}=\sum_{y=1}^{q N-1} \frac{T_{y}}{y(y+1)}
$$

where $T_{y}=\sum_{k=1}^{y} \sin \left(2 \pi \frac{x k}{N}\right)$.

Lemma 2 follows from Abel transformation and $T_{q N}=0$.

LEMma 3. For any positive integers $q, N$ we have

$$
S=\sum_{x=1}^{N} \sum_{y=1}^{q N} \frac{\sin \left(2 \pi \frac{x y}{N}\right)}{x y}>c_{1} \log N-c_{2}
$$

Proof. As $\sum_{1 \leq y \leq Y} \sin (\alpha y) / y=O(1)$ uniformly in $\alpha$ and $Y$, by Lemma 2 we have

$$
S=\sum_{1 \leq x \leq N / 8} x^{-1} \sum_{y=1}^{q N} \frac{T_{y}}{y(y+1)}+O\left(\sum_{N / 8<x \leq N} x^{-1}\right)
$$

where

$$
T_{y}=\sum_{k=1}^{y} \sin \left(2 \pi \frac{x k}{N}\right)=\frac{\sin ^{2}\left(\pi \frac{x}{N} y\right) \cos \left(\pi \frac{x}{N}\right)}{\sin \left(\pi \frac{x}{N}\right)}+\frac{1}{2} \sin \left(2 \pi \frac{x y}{N}\right) .
$$

Using again $\sum_{1 \leq x \leq N / 8} \sin (\alpha x) / x=O(1)$ we have

$$
S>\sum_{1 \leq x \leq N / 8} x^{-1} \sum_{1 \leq y \leq N /(2 x)} \frac{\sin ^{2}\left(\pi \frac{x}{N} y\right) \cos \left(\pi \frac{x}{N}\right)}{y(y+1) \sin \left(\pi \frac{x}{N}\right)}-O(1) .
$$

The ranges of variables are such that

$$
\sin ^{2}\left(\pi \frac{x}{N} y\right) \geq \frac{4 x^{2} y^{2}}{N^{2}}, \quad \cos \left(\pi \frac{x}{N}\right) \geq \frac{1}{2}, \quad 0<\sin \left(\pi \frac{x}{N}\right)<\pi \frac{x}{N}
$$

Therefore

$$
S>\sum_{1 \leq x \leq N / 8} x^{-1} \sum_{1 \leq y \leq N /(2 x)} \frac{x}{\pi N}-O(1)>c_{1} \log N-c_{2} .
$$

Lemma 3 is proved. 
3. Proof of the Theorem. We put

$$
\alpha=2 \pi \sum_{n=1}^{\infty} \frac{1}{q_{n}}
$$

where the sequence $q_{1}, q_{2}, \ldots$ is defined as follows: $q_{1}=2, q_{n+1}=q_{n}^{n q_{n}+1}$ for all positive integers $n$. In order to prove the Theorem it is enough to establish that for this $\alpha$ the subsequence $h_{q_{n}}$ of (1) diverges as $n \rightarrow \infty$.

For a given $n$ set $q=q_{n}, N=q_{n}^{n q_{n}}$. We will prove that

$$
h_{q_{n+1}}=h_{q N}>c_{1} q^{-1} \log N-c_{2} \log q>c n
$$

for all large enough $n$.

Since

$$
\alpha=2 \pi\left(\frac{a}{q}+\frac{1}{q N}\right)+O\left(\frac{1}{q^{3} N^{3}}\right)
$$

we have

$$
h_{q_{n+1}}=\sum_{x=1}^{q N} \sum_{y=1}^{q N} \frac{\sin \left(2 \pi\left(\frac{a}{q}+\frac{1}{q N}\right) x y\right)}{x y}+O(1) .
$$

Put

$$
S_{1}=\sum_{x \leq q N}^{\prime} \sum_{y=1}^{q N} \frac{\sin \left(2 \pi\left(\frac{a}{q}+\frac{1}{q N}\right) x y\right)}{x y}
$$

and

$$
S_{2}=\sum_{x \leq q N}^{\prime \prime} \sum_{y=1}^{q N} \frac{\sin \left(2 \pi\left(\frac{a}{q}+\frac{1}{q N}\right) x y\right)}{x y}
$$

where the prime indicates the additional condition $x \equiv 0(\bmod q)$ while two primes mean $x \not \equiv 0(\bmod q)$.

We have

$$
h_{q_{n+1}}=S_{1}+S_{2}+O(1) .
$$

From Lemma 3 it follows that $S_{1}>c_{1} q^{-1} \log N-c_{2}=c_{1} n \log q-c_{2}$, i.e.

$$
S_{1}>c_{1} n \log q-c_{2} \text {. }
$$

Now we prove that $S_{2}>-c_{3} \log q$.

The subsum of $S_{2}$ over $0<x<q$ is $O(\log q)$. Therefore according to Lemma 1 we have

$$
S_{2}=\pi S_{3}+\pi S_{4}+O(\log q)
$$


where

$$
\begin{gathered}
S_{3}=\sum_{q<x<q N}^{\prime \prime} x^{-1} \varrho\left(\frac{a x}{q}+\frac{x}{q N}\right), \\
S_{4}=\sum_{q<x<q N}^{\prime \prime} x^{-1} O\left(\frac{1}{1+q N\left|\sin \pi\left(\frac{a x}{q}+\frac{x}{q N}\right)\right|}\right) .
\end{gathered}
$$

Further, since $x^{-1} O(f)=O\left(x^{-2}\right)+O\left(f^{2}\right)$, we have

$$
S_{4}=O(1)+O\left(\sum_{x=1}^{q N} \frac{1}{1+q^{2} N^{2} \sin ^{2} \pi\left(\frac{a x}{q}+\frac{x}{q N}\right)}\right) .
$$

Taking into account that $q$ and $N$ are powers of 2 we see that when $x$ runs through a complete system $(\bmod q N)$, then so does $(a N+1) x$. Hence

$$
\begin{aligned}
S_{4} & =O(1)+O\left(\sum_{x=1}^{q N} \frac{1}{1+q^{2} N^{2} \sin ^{2} \frac{\pi x}{q N}}\right) \\
& =O(1)+O\left(\sum_{x=1}^{q N / 2} \frac{1}{1+q^{2} N^{2} \sin ^{2} \frac{\pi x}{q N}}\right) .
\end{aligned}
$$

Using the fact that

$$
\sin \frac{\pi x}{q N}>\frac{x}{q N} \quad \text { for } 1 \leq x \leq q N / 2
$$

we obtain

$$
S_{4}=O(1)
$$

In order to estimate $S_{3}$ we note that

$$
\left\{\frac{a x}{q}+\frac{x}{q N}\right\} \leq\left\{\frac{a x}{q}\right\}+\left\{\frac{x}{q N}\right\}=\left\{\frac{a x}{q}\right\}+\frac{x}{q N} .
$$

Therefore

$$
S_{3} \geq \sum_{q<x<q N}^{\prime \prime} x^{-1}\left(\varrho\left(a x q^{-1}\right)-\frac{x}{q N}\right)>-1+\sum_{q<x<q N}^{\prime \prime} x^{-1} \varrho\left(a x q^{-1}\right) .
$$

Substitution $x=q u+l$ where $1 \leq u \leq N-1,1 \leq l \leq q-1$ gives us

$$
\begin{aligned}
1+S_{3} & >\sum_{u=1}^{N-1} \sum_{l=1}^{q / 2-1}(q u+l)^{-1} \varrho\left(a l q^{-1}\right)+\sum_{u=1}^{N-1} \sum_{l=q / 2+1}^{q-1}(q u+l)^{-1} \varrho\left(a l q^{-1}\right) \\
& =\sum_{u=1}^{N-1} \sum_{l=1}^{q / 2-1}(q u+l)^{-1} \varrho\left(a l q^{-1}\right)+\sum_{u=1}^{N-1} \sum_{l=1}^{q / 2-1}(q u+q-l)^{-1} \varrho\left(-a l q^{-1}\right)
\end{aligned}
$$

where we have used $\varrho(1 / 2)=0$. 
Now note that $\varrho\left(-a l q^{-1}\right)=-\varrho\left(a l q^{-1}\right)$. Therefore

$$
1+S_{3}>-\sum_{u=1}^{N-1} \sum_{l=1}^{q / 2-1}\left(\frac{1}{q u+l}-\frac{1}{q u+q-l}\right)>-\sum_{u=1}^{N-1} \sum_{l=1}^{q / 2-1} \frac{q}{q^{2} u^{2}}>-2,
$$

whence $S_{3}>-3$. Together with (5), (4), (3), (2) and $q=q_{n}$ we obtain

$$
h_{q_{n+1}}>c_{1} n \log q_{n}-c_{4} \log q_{n}-c_{5} .
$$

The Theorem is proved.

Acknowledgements. The author would like to thank the referee for the corrections.

\section{References}

[1] G. I. Arkhipov and K. I. Oskolkov, On special trigonometric series and its application, Math. USSR-Sb. 62 (1989), 145-155.

[2] G. I. Arkhipov, V. A. Sadovnichii and V. N. Chubarikov, Lectures in Mathematical Analysis, Vysshaya Shkola, Moscow, 1999 (in Russian).

[3] K. I. Oskolkov, Vinogradov series and integrals and their applications, Proc. Steklov Inst. Math. 190 (1992), 193-229.

[4] - Vinogradov series in the Cauchy problem for equations of Schrödinger type, ibid. 200 (1993), 291-315.

Institute of Mathematics

Academia Sinica

Taipei 11529, Taiwan

E-mail: garaev@math.sinica.edu.tw

Received on 28.5.2001

and in revised form on 30.7.2001 